\title{
What is the biological basis of sensorimotor integration?
}

\author{
Martha Flanders
}

Received: 18 July 2010 / Accepted: 29 December 2010 / Published online: 2 February 2011

(C) The Author(s) 2011. This article is published with open access at Springerlink.com

\begin{abstract}
This Prospects presents the problems that must be solved by the vertebrate nervous system in the process of sensorimotor integration and motor control. The concepts of efference copy and inverse model are defined, and multiple biological mechanisms are described, including those that form the basis of integration, extrapolation, and comparison/cancellation operations. Open questions for future research include the biological basis of continuous and distributed versus modular control, and somatosensory-motor coordination.
\end{abstract}

Keywords Motor control - Somatosensory - Haptic . Sensory feedback · Efference copy · Forward model

\section{Introduction}

Over the last few decades, a great deal of research effort has been devoted to gaining a better understanding of the neural control of movement. In the 1970s, engineering control systems approaches allowed physiologists to get a handle on processes involving feedback, such as smooth pursuit, where the input is movement (slip) of a target across the retina and the output is an eye movement that negates this retinal slip. In the 1980s and 1990s, these engineering advances in the oculomotor field gradually evolved into a nearly complete understanding of saccadic eye movements. This was accomplished by incorporating, into what originally began as an engineering approach, neural recordings from subcortical structures, and neurobiological concepts such as the alignment of sensory and motor maps in the superior colliculus. However, at the

M. Flanders $(\varangle)$

Department of Neuroscience, University of Minnesota, Minneapolis, MN 55455, USA

e-mail: fland001@umn.edu turn of the century, decade 2000 seemed to host a redirection of research focus, away from basic biological mechanisms and toward issues such as which sensorimotor correspondences can be learned and remembered, and how best to decode neural information for prosthetic applications.

While learning is always a topic of interest, and it is important to translate neurobiological research results into advances in prosthetics, the purpose of this opinion paper is to return attention to questions about biological mechanism. First, I will list the basic physiological processes that must be implemented by the nervous system in sensorimotor integration. Then, I will show that these processes can be best understood in light of the types of biological operations that are known to be available to the system. I will give examples of these biological operations and then I will give examples of lines of investigation that, in my opinion, have not helped in answering questions about the neurobiology of sensorimotor integration. Finally, I will suggest more fruitful approaches for the future.

\section{Problems that must be solved by the nervous system}

\subsection{Interpreting sensory targets}

All sensory input is filtered by the nervous system according to its destination and use. If the use is perception, the filtering may take the form of feature extraction and combination of inputs from different sensory sources, such as the integration of visual and auditory information. When sensory input is instead used to instruct the generation of motor commands, it must be interpreted in terms of the current state of the motor system. For example, when the visual system provides a target position that requires an arm movement, the motor command must be appropriate for the hand's initial position. Obviously, to move the hand in the mid-sagittal 


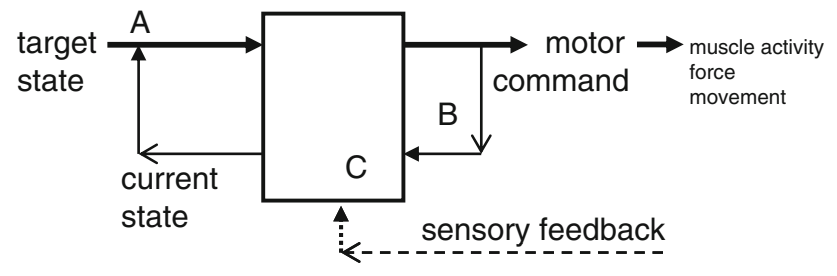

Fig. 1 A diagram of the general process of transforming target information into motor commands for movements. $A$ The current state of the system is subtracted from the target state to create a motor error, or desired movement signal, which is then used to produce a motor command to the muscles. $B$ Efference copy is used to keep track of the expected result of the motor command, and this is used to update the current state. $C$ Delayed sensory feedback is used to update the association (box) between the desired movement signal and the motor command

plane, motor commands and muscle activations would differ depending on whether the hand began in front of, or beyond, the visual target. Therefore, the first step in the process must be to re-express the neural representation of the target state, in terms of the difference between the current state and the target state (Fig. 1A).

\subsection{Employing efference copy}

The needed representation of current state could potentially be provided by sensory systems. However, using sensory input sometimes takes too much time. Therefore, to keep track of the current state, the system appears to use a form of internal monitoring called efference copy (Fig. 1B). Efference copy simply means an internal copy of outgoing information. It is a term for the idea that the motor system forms a representation of its own current state, here used as a baseline for information about the target state (Fig. 1A). This classic concept was fully described by von Holst and Mittelstaedt (1950) with examples from a wide variety of motor behaviors, ranging from orienting movements in fish and insects, to spinal reflexes in mammals.

\subsection{Using memory as a filter}

Memories may be old ones that have been previously stored by synaptic modifications, or new ones that are continuously updated and maintained by physiological activity. Sensory feedback can be used to modify the old memories, thus forming new memories. For example, an incorrect estimation of the mechanical properties of a hand-held object is sensed and then corrected, and the corrected mechanical properties are stored in memory. As indicated in Fig. 1C, the correction may be based on a comparison between the sensory state that is associated with the efference copy of the motor command (Fig. 1B) and the true sensory state, as indicated by the delayed sensory feedback (dashed arrows).

\subsection{Producing motor commands}

Thus, as diagramed in Fig. 1 (thick solid arrows), subtracting the current state from the target state, and filtering this signal through memory, results in the motor commands that give rise to muscle activation, force, and movement. This filtering is essentially a transformation from sensory (movement) parameters to motor (force) parameters, the inverse of the causal relation of force to movement, and the memory filter has been referred to as the "inverse model." As mentioned above, there has been a great deal of research on what sensorimotor correspondences can be stored in the inverse model. There has also been an ongoing debate about how the motor command maps into muscle activity. Before discussing these issues, we will first consider some of the biological operations that are available to the system to solve problems like these.

\section{Neural filtering mechanisms}

\subsection{Feature extraction}

The series of neuronal activations/inhibitions that is associated with sensation can be characterized as a process of feature extraction. For example, in the visual system, neurons may be connected to one another in a manner that makes them particularly sensitive to certain features of the visual image. As cartooned in Fig. 2a, the neural network, through genetic and activity dependent mechanisms, may establish weighting coefficients $(a, b, c)$ to accentuate certain features of the input (the circle) and downplay others (e.g., the rectangles). Importantly, this simple example highlights the fact that most neural operations are accomplished through the distributed activity of groups of interconnected neurons, with little evidence of distinct modularity.

\subsection{Integrating, differentiating, and extrapolating}

Other key features of neural processing are phenomena similar to low-pass and high-pass filtering. When action potentials are recorded from neurons in the sensorimotor system, these spike trains are often characterized as tonic or phasic, and the neurons are then classified as slowly adapting (low-pass) or rapidly adapting (high-pass), respectively. A neuron that responds to a transient input with a sustained discharge can form the basis for neural integration. Conversely, a neuron can be regarded as a high-pass filter if it responds transiently to a change in the level of a maintained input; this neuron may be sensitive to the time derivative of a signal (such as velocity rather than position).

In the vestibular sensory system, there has been some debate about whether neural integration is a cellular 
A. Extraction

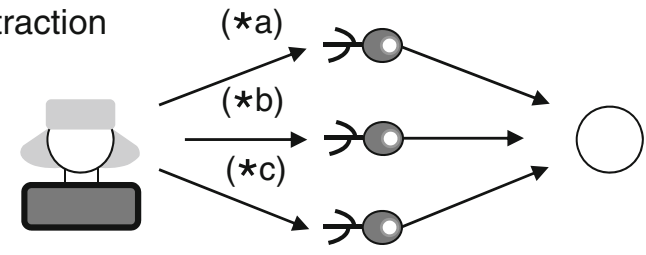

B. Extrapolation
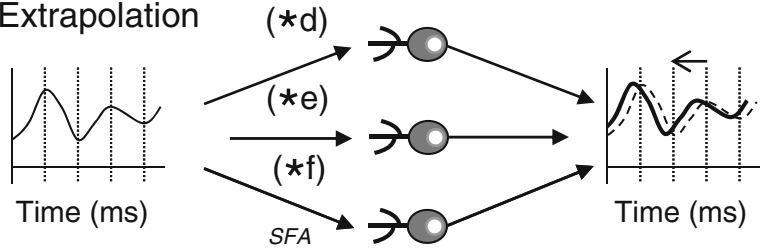

STD

Fig. 2 Two types of biological filtering operations: a feature extraction, and $\mathbf{b}$ extrapolation of a smoothly changing sensory signal forward in time. In each case, the input/output associations are made by establishing weighting coefficients $(a-f)$ at individual synapses. The learning might also involve intrinsic cell properties such as spike frequency adaptation (SFA) and short-term depression (STD)

phenomenon or a network function or both. The vestibular semicircular canals use fluid inertia and tissue stiffness to create a sensory signal proportional to head rotational velocity. Thus, the sensory input from the semicircular canals is transient during maintained rotations. However, a network of neurons involving the vestibular nucleus integrates its head velocity-related inputs to provide the signal used to control eye-in-head position (Cannon et al. 1983). A recent experimental and modeling study indicated that this mathematical integration is accomplished through a combination of several biological mechanisms (Aksay et al. 2007). First, consistent with the Robinson model (Arnold and Robinson 1991), the system appears to take advantage of the push/pull opponency between left and right ear inputs to form a bilateral positive feedback system based on inhibition. Furthermore, there is evidence for recurrent network activity on each side, to help maintain the activity initiated by excitatory phasic inputs. Finally, intrinsic cellular mechanisms such as plateau potentials may allow individual neurons to integrate synaptic inputs. Although not as fully studied, it appears that similar mechanisms may allow for integration of somatosensory input for memory operations (Romo et al. 1999), and signals related to navigation for path integration (Taube and Bassett 2003).

Whereas the use of multiple mechanisms for neural integration is relatively well established, neural differentiation seems a bit more controversial and unresolved (Krekelberg and Lappe 2002). In visual motion processing, it is desirable to make up for time delays by extrapolating the target trajectory. This could potentially be done using phasic, velocity-related signals. Classic research (Pellionisz and Llinas 1979) suggested that neural circuits essentially implement a Taylor series expansion, combining position- and velocity-related inputs to output a signal related to the position expected at a future time. In more recent work, Puccini et al. (2007) showed that this velocity sensitivity and the associated phase advance could be achieved with a combination of spike frequency adaptation and short-term synaptic depression (SFA and STD in Fig. 2b). Interestingly, this brain slice recording and simulation study showed that adding SFA to STD decreased presynaptic firing rates to reduce postsynaptic fluctuations, thus boosting the phase lead while also reducing the random variability in the output signal.

Alternatively, or perhaps in addition, extrapolation of visual target position could be a network operation. A neural network trained with smooth target trajectories (weighting coefficients $d, e, f$, in Fig. 2b) could transform current target position into the future target position that usually results from this type of familiar trajectory. A modeling study by Kawato and colleagues (Shibata et al. 2005) demonstrated that this is potentially an operation performed by neurons in visual cortical area MST. As is the case for neural integration, it seems possible that differentiation is achieved through a combination of intrinsic cellular and network operations.

\subsection{Comparison and cancellation}

There are two classic examples of cases where inputs to particular groups of neurons are known to be either added or subtracted to perform the behavioral functions of comparison and cancellation, respectively. The first is illustrated in Fig. 3a, which schematically shows neurons in an avian brainstem auditory nucleus getting convergent input from the two ears. The incoming action potential spike trains are phaselocked to the sound wave and due to the time it takes for axons to conduct action potentials, a sound that first enters the right ear causes action potentials to reach some neurons sooner than others. In the barn owl, the anatomical arrangement of the axons creates the proper pattern of delay to allow the neurons to act as coincidence detectors (Carr and Konishi 1990). This allows the system to use timing signals to encode the relative right/left location of the sound source. A more complex type of binaural convergence can allow the system to combine sound intensities between the two ears (e.g., Park et al. 1997).

As a second classic example of a system using a convergence of inputs, the electrosensory lateral line nucleus of some mormyrid fish is known to perform a filtering operation more akin to cancellation. Another, nearby brainstem nucleus provides a motor command to the electric organ and, in electrophysiological experiments, an efference copy of this command was detected in the electrosensory nucleus (Bell 1982). The incoming sensory signal and the efference copy had a subtractive interaction in the nucleus, which could allow the 


\section{A. Comparison}

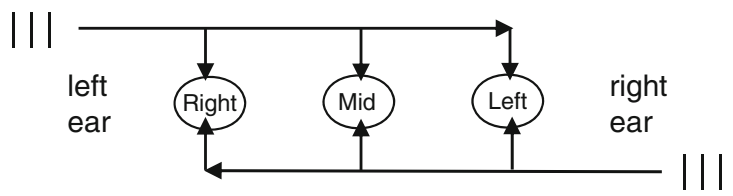

B. Cancellation

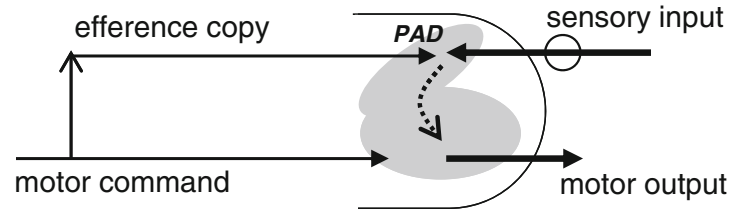

Fig. 3 Examples of biological mechanisms that might be used to combine signals: a comparison, and $\mathbf{b}$ cancellation. In the avian auditory system, $\mathbf{a}$ inputs from the two ears are known to be combined for sound localization. In the somatosensory system, $\mathbf{b}$ it is hypothesized that the efference copy may be used to cancel the sensory input that would be expected to result from the motor command. This may involve presynaptic inhibition of cutaneous afferent input (primary afferent depolarization, PAD), as shown in more detail in Fig. 4a

fish to detect electric field patterns that are different from those produced by its own discharge.

A more recent example of this sort of cancellation is shown in Fig. 3b. It is well known that the corticospinal tract projects from motor cortex to the ventral horn of the spinal cord, carrying the motor command to the motoneurons. However, a lesser known projection of the corticospinal tract is to the dorsal part of the spinal cord, where it converges with incoming input from somatosensory receptors. This may serve as an efference copy system to cancel the somatosensory input predictably produced by the associated motor command (Lemon 2008). Seki et al. $(2003,2009)$ showed that this filtering of "expected" somatosensory feedback may be accomplished by primary afferent depolarization (PAD), where signals arising from descending motor commands can selectively reduce firing rates in the intraspinal terminals of muscle spindle, tendon organ, or cutaneous afferents (Fig. 4a, see Rudomin and Schmidt 1999).

This sort of cancellation of somatosensory input can also occur in the brain (Fig. 4b). Both the somatosensory cortex and the motor cortex send corticofugal projections to the dorsal column nuclei in the medulla, an important component of the afferent somatosensory pathway. Although the exact cellular mechanisms are yet to be determined, the net effect of this corticofugal projection appears to be inhibition of the somatosensory input associated with voluntary arm and hand movements (Towe and Jabbur 1961; Shin and Chapin 1989).

The results of a recent psychophysical study (Weiss and Flanders 2011) are consistent with the hypothesis that this
A. Spinal Cord

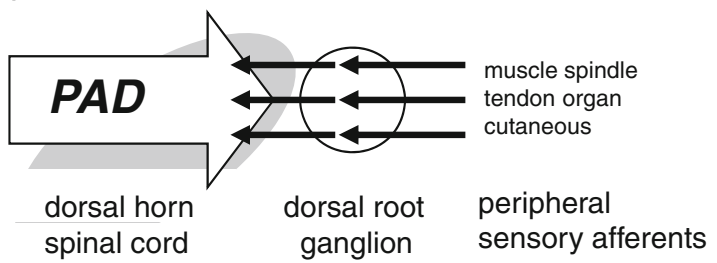

B. Brain

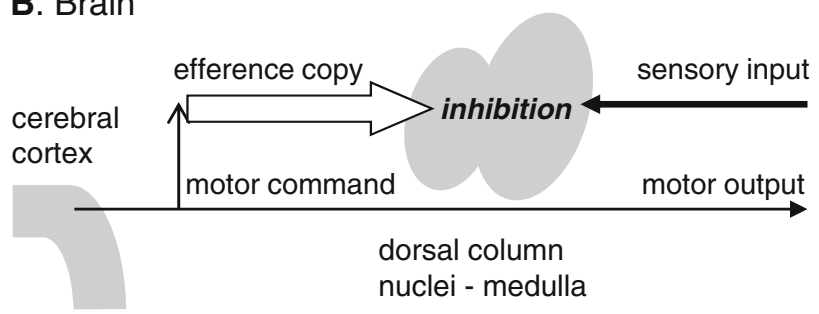

Fig. 4 Possible sites of inhibition of cutaneous afferent input in vertebrates. a Spinal interneurons reduce the efficacy of synaptic transmission by synapsing on the intraspinal terminals of afferent fibers from various types of peripheral somatosensory receptor. b Corticofugal projections originating in motor cortex tend to inhibit incoming somatosensory signals in the dorsal column nuclei

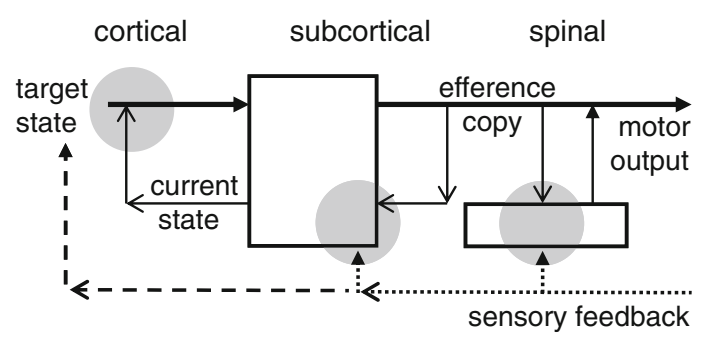

Fig. 5 Sensory filtering at cortical, subcortical, and spinal levels. The gray circles highlight areas of hypothesized neural comparison/cancellation operations, involving activity resulting from efference copy and sensory feedback

cancellation mechanism is used during haptic exploratory movements. When human subjects ran the index finger along a curved surface, they reacted to a change from the expected curvature with a compensatory decrease in the activity of the stretched muscles, at a spinal reflex latency. This response was opposite to the well-known stretch reflex, but consistent with a hypothetical involvement of an afferent inhibitory mechanism. We proposed that this sort of somatosensory comparison/cancellation is a fundamental neural operation that takes place at multiple levels: the cerebral cortex, subcortical structures (including the cerebellum and dorsal column nuclei), and the spinal cord. As illustrated in Fig. 5, the current state or the sensory input that is expected based on efference copy is essentially subtracted from sensory feedback to drive an ongoing or corrective movement. 


\section{Research in computational motor control}

\subsection{Bayesian inference}

As outlined in Figs. 1 and 5, basic sensorimotor operations must rely upon the generation of an expected sensory state, which is a learned association between copies of previous motor commands and their resulting sensory states. This expectation based on prior activities is then combined with new information about the true sensory state. Thus, at first glance, it appears that the central nervous system is implementing a Kalman filter or performing a process akin to Bayesian inference, where probabilities derived from prior events are combined with newly sensed parameters. However, while probabilistic statistical methods have been useful in prosthetic decoding algorithms (e.g., Wu et al. 2006) and in predicting behavioral decisions (e.g., Kim and Basso 2010), this approach is not typically directed toward revealing the biological basis of sensorimotor processing. Hopefully, future studies will elucidate the cellular mechanisms used to bias neural output based on the reliability of recent memories.

\subsection{Inverse models}

Compared to the recently popular use of probabilistic analytical tools, studies of the process of internal model learning might seem to be further along in explaining neural operations. As mentioned above, the inverse internal model can be defined as a memory filter used in the transformation from kinematic parameters (e.g., velocity) to kinetic parameters (e.g., force). Numerous studies on this topic have been conducted (see Cisek 2009 for a review). It has been concluded that learning is sometimes in the frame of reference of the body rather than the world, kinematic and kinetic parameters may or may not be learned independently depending on the situation, and impedance (stiffness) is learned by a different process. In one interesting study, Thoroughman and Shadmehr (2000) showed that learning new forces for one movement direction influences movements in neighboring directions in a manner reminiscent of the breadth of directional sensitivities of neurons in the motor system.

One might suppose that this active field of research on inverse models would inspire and direct current research in neuroprosthetics, but this does not seem to be the case. Instead some of the most successful neuroprosthetic studies capitalize on the classic observation of Fetz and Finocchio (1975) that motor cortical neurons exhibit remarkable plasticity. For example, by recording from the same set of identified neurons for multiple days, Ganguly and Carmena (2009) showed that motor cortical neurons could be trained to represent randomly chosen new associations between their activities and the movement of a computer cursor.

\subsection{Modularity}

As mentioned above with regard to Fig. 2, from a biological perspective, sensorimotor operations are expected to involve numerous interconnected neurons. Contrary to this assumption, a currently active area of motor control research is based on the premise of the existence a small number of discrete motor control modules, called motor primitives or muscle synergies (see d'Avella 2009 for a review). A muscle synergy is defined as a group of muscles that work together, although experimental work suggests that for primate arm and hand muscles this should be redefined as a multi-muscle group of motor units that work together (Herrmann and Flanders 1998; Weiss and Flanders 2004).

Characterizing a complex set of data by identifying its basis functions, or primitives, is clearly a useful approach. For example, our group showed that a wide range of human hand shapes could be well reconstructed as the weighted sum of just a few patterns of covariation of joint angles (Santello et al. 1998), and this discovery has been used in prosthetic/robotic applications (Ciocarlie et al. 2007). We also showed that an even wider range of human hand shapes and movements could be represented by a small number of muscle synergies (Weiss and Flanders 2004; Klein Breteler et al. 2007).

However, these observations should not be interpreted as support for the premise of discrete modularity. To the contrary, the hand shape and the muscle synergy basis functions described a distributed, not modular, organization: Santello et al. (1998) showed that numerous higher order principal components of hand shapes represented important features of the data set, not random variability, and our subsequent studies showed that each additional muscle synergy provided a smoothly increasing amount of variance accounted for. It is not clear what would be the biological advantage of control with a small number of discrete motor control modules, instead of distributed control.

An elegant simulation article by Chhabra and Jacobs (2006) addressed this question. These authors demonstrated that the existence and advantage of a small number of basis functions/muscle synergies was at the level of computational theory, which is distinct from the level of hardware implementation (see Marr 1982). Furthermore, a computational analysis by Lewicki and Sejnowski (2000) suggested that distributed, "overcomplete" basis functions, in addition to being inherently more neurobiological, can provide highly efficient solutions to reconstruction problems such as speech generation. 


\section{More open questions}

\subsection{What is the biological basis of efference copy?}

The idea that the sensorimotor system must use efference copy is well accepted (reviewed by Crapse and Sommer (2008), Poulet and Hedwig (2007)). In some invertebrate examples, the anatomical basis has been established (Poulet and Hedwig 2006; Webb 2004), but in many other cases efference copy still tends to be an amorphous concept. As initially described by von Holst and Mittelstaedt 1950, the idea can be readily applied to automatic systems such as oculomotor and spinal reflexes, and the field of spinal cord/locomotor research is close to unraveling the complex neural substrates (Rudomin and Schmidt 1999; Grillner and Jessell 2009).

As illustrated in Fig. 1, efference copy has two main functions: (1) to give rise to a real-time estimate of current state, and (2) to allow a comparison with delayed sensory feedback. We can ask more specific questions about each aspect:

(1) How does the system represent its current state? In oculomotor control, the current eye position is constantly represented by the pattern of activity in the motor nuclei. For more complex movements, however, the relation between motor neuronal firing frequencies and effector position is not one to one, and state estimation may rely on the current pattern of activity in the somatosensory system. Johansson and Flanagan (2009) proposed that sequences of hand and arm movement are broken into discrete segments so that particular somatosensory events can trigger the next "action phase." This would correspond to the longest dashed feedback loop in Fig. 5. However, fluent hand movements sometimes involve significant blending of one segment into the next (Jerde et al. 2003), suggesting that current state must be continuously represented and used to tailor ongoing actions (the efference copy/current state feedback loop in Fig. 5).

(2) How is sensory feedback evaluated? As mentioned above, we have hypothesized that a cancellation mechanism is used during haptic exploration (Weiss and Flanders 2011). However, the locations and forms of this cancellation are yet to be determined. In general, active sensing must involve some sort of comparison or cancellation, and this phenomenon is often referred to as sensory gating (reviewed by Chapman (2009)). The biological implementation of this type control algorithm has yet to be determined, although clear progress is being made in studies of rat whisker movements (e.g., Hentschke et al. 2006).

\subsection{To what extent is sensory input extrapolated?}

The best examples of target trajectory extrapolation come from research on the visual system (e.g., Berry et al. 1999). Collectively, this research has shown that derivative operations are basic biological phenomena that occur at many different sites (from retina to cortex) and via many different mechanisms (from cellular adaptation to network operations). However, more work is needed to parameterize this process for the visuomotor system (e.g., Soechting et al. 2010). For example, what is the time delay of the visual signal in the smooth pursuit eye movement system under various conditions? It seems reasonable to assume that similar extrapolation processes are available to the auditory and somatosensory systems, but much less is known.

\subsection{Can hardware models help solve these problems?}

There are several types of approach to analysis, modeling, and simulation. One possibility is to build a hardware model, such as a robot programmed to perform a neural function in the same manner that the nervous system is thought to do so (reviewed by Pfeifer et al. 2007). A recent success in the field of active sensing is robots programmed to gather and interpret the same sensory parameters as rat whiskers (Solomon and Hartmann 2006; Fox et al. 2009). Numerous other issues could be approached. For example, a tendency in robotic research is to break a task into modules, but perhaps it would be possible to explicitly test whether a more continuous, distributed control strategy would be advantageous in some situations. Although ideal robotic and human solutions are not necessarily the same, robotic research potentially provides a suitable testing ground to quantify the relative merits of different biological control algorithms.

\subsection{Which questions are appropriate for simulation?}

Sensorimotor research inherently focuses on processes that involve multiple parameters and multiple processing algorithms. For example, an important unexplored issue is the interplay between somatosensory and motor systems. Even at the periphery, these systems are intertwined. For example, muscle spindle sensory receptors contain tiny muscles! Other somatosensory receptors also respond differently depending on the actively generated state of the surrounding musculature. How are inputs from the multiple types of somatosensory receptors evaluated in light of their active versus passive components, and how are they combined? How can this somatosensory signal be extrapolated forward in time to make up for sensory processing delays? How does this representation interact with the representation of motor commands? These complex questions will require multiple experimental approaches, undoubtedly including computer simulation. 
Acknowledgment Supported by the National Institute of Neurological Disorders and Stroke R01 NS027484. The author thanks Professors John Soechting and Randy Nelson.

Open Access This article is distributed under the terms of the Creative Commons Attribution Noncommercial License which permits any noncommercial use, distribution, and reproduction in any medium, provided the original author(s) and source are credited.

\section{References}

Aksay E, Olasagasti I, Mensh BD, Baker R, Goldman MS, Tank DW (2007) Functional dissection of circuitry in a neural integrator. Nat Neurosci 10:494-504

Arnold DB, Robinson DA (1991) A learning network model of the neural integrator of the oculomotor system. Biol Cybern 64:447-454

Bell CC (1982) Properties of a modifiable efference copy in an electric fish. J Neurophysiol 47:1043-1056

Berry MJ II, Brivanlou IH, Jordan TA, Meister M (1999) Anticipation of moving stimuli by the retina. Nature 398:334-338

Cannon SC, Robinson DA, Sharma SA (1983) A proposed neural network for the integrator of the oculomotor system. Biol Cybern 49:127-136

Carr CE, Konishi M (1990) A circuit for detection of interaural time differences in the brain stem of the barn owl. J Neurosci 10:32273246

Chapman CE (2009) Active touch. In: Binder MD, Hirokawa N, Windhorst U (eds) Encyclopedia of neuroscience. Springer, Berlin pp 35-41

Chhabra M, Jacobs RA (2006) Properties of synergies arising from a theory of optimal motor behavior. Neural Comput 18:2320-2342

Ciocarlie M, Goldfeder C, Allen PK (2007) Dimensionality reduction for hand-independent dexterous robotic grasping. International conference on intelligent robots and systems, San Diego

Cisek P (2009) Internal models. In: Binder MD, Hirokawa N, Windhorst U (eds) Encyclopedia of neuroscience. Springer, Berlin pp 2010-2112

Crapse TB, Sommer MA (2008) Corollary discharge across the animal kingdom. Nat Rev Neurosci 9:587-600

d'Avella A (2009) Muscle synergies. In: Binder MD, Hirokawa N, Windhorst U (eds) Encyclopedia of neuroscience. Springer, Berlin pp 2509-2512

Fetz EE, Finocchio DV (1975) Correlations between activity of motor cortex cells and arm muscles during operantly conditioned response patterns. Exp Brain Res 23:217-240

Fox CW, Mitchinson B, Pearson MJ, Pipe AG, Prescott TJ (2009) Behavioral dependency of texture classification in a whiskered mobile robot. Auton Robots 26:223-239

Ganguly K, Carmena JM (2009) Emergence of a stable cortical map for neuroprothetic control. PLOS Biol 77:e1000153

Grillner S, Jessell TM (2009) Measured motion: searching for simplicity in spinal locomotor networks. Curr Opin Neurobiol 19:572-586

Hentschke H, Haiss F, Schwarz C (2006) Central signals rapidly switch tactile processing in rat barrel cortex during whisker movements. Cereb Cortex 16:1142-1156

Herrmann U, Flanders M (1998) Directional tuning of single motor units. J Neurosci 18:8402-8416

Jerde TE, Soechting JF, Flanders M (2003) Coarticulation in fluent fingerspelling. J Neurosci 23:2383-2393

Johansson RS, Flanagan JR (2009) Coding and use of tactile signals from the fingertips in object manipulation tasks. Nat Rev Neurosci $10: 345-359$
Kim B, Basso MA (2010) A probabilistic strategy for understanding action selection. J Neurosci 30:2340-2355

Klein Breteler MD, Simura KJ, Flanders M (2007) Timing of muscle activation in a hand movement sequence. Cereb Cortex 17:803815

Krekelberg B, Lappe M (2002) Neuronal latencies and the position of moving objects. Trends Neurosci 24:335-339

Lemon RN (2008) Descending pathways in motor control. Ann Rev Neurosci 31:195-218

Lewicki MS, Sejnowski TJ (2000) Learning overcomplete representations. Neural Comput 12:337-365

Marr D (1982) Vision. W.H. Freeman and Co, San Francisco

Park TJ, Monsivais P, Pollak GD (1997) Processing of interaural intensity differences in the LSO: role of interaural threshold differences. J Neurophysiol 77:2863-2878

Pellionisz A, Llinas R (1979) Brain modeling by tensor network theory and computer simulation. The cerebellum: distributed processor for predictive coordination. Neuroscience 4:323-348

Pfeifer R, Lungarella M, Iida F (2007) Self-organization, embodiment, and biologically inspired robotics. Science 318:1088-1093

Poulet JFA, Hedwig B (2006) The cellular basis of a corollary discharge. Science 311:518-522

Poulet JFA, Hedwig B (2007) New insights into corollary discharges mediated by identified neural pathways. Trends Neurosci 30:14 21

Puccini GD, Sanchez-Vives MV, Compte A (2007) Integrated mechanisms of anticipation and rate-of-change computations in cortical circuits. PLOS Comput Biol 3:813-825

Romo R, Brody CD, Hernandez A, Lemus L (1999) Neuronal correlates of parametric working memory in prefrontal cortex. Nature 399:470-473

Rudomin P, Schmidt RF (1999) Presynaptic inhibition in the vertebrate spinal cord revisited. Exp Brain Res 129:1-37

Santello M, Flanders M, Soechting JF (1998) Postural hand synergies for tool use. J Neurosci 18:10105-10115

Seki K, Perlmutter SL, Fetz EB (2003) Sensory input to primate spinal cord is presynaptically inhibited during voluntary movement. Nat Neurosci 6:1309-1316

Seki K, Perlmutter SL, Fetz EB (2009) Task-dependent modulation of primary afferent depolarization in cervical spinal cord of monkeys performing an instructed delay task. J Neurophysiol 102:85-99

Shibata T, Tabata H, Schaal S, Kawato M (2005) A model of smooth pursuit based on learning target dynamics. Neural Netw 18:213224

Shin H-C, Chapin JK (1989) Mapping the effects of motor cortex stimulation on single neurons in the dorsal column nuclei in the rat: direct responses and afferent modulation. Brain Res Bull 22:245252

Soechting JF, Rao HM, Juveli JZ (2010) Incorporating prediction in models for two-dimensional smooth pursuit. PLoS ONE 5(9):e12574

Solomon JH, Hartmann MJ (2006) Robotic whiskers used to sense features. Nature 443:525

Taube JS, Bassett JP (2003) Persistent neural activity in head direction cells. Cereb Cortex 13:1162-1172

Thoroughman KA, Shadmehr R (2000) Learning of action through adaptive combination of motor primitives. Nature 407:742-747

Towe AL, Jabbur SJ (1961) Cortical inhibition of neurons in dorsal column nuclei of cat. J Neurophysiol 24:488-498

von Holst E, Mittelstaedt H (1950) Das Reafferenzprinzip. Naturwissenschaften 37:464-476

Webb B (2004) Neural mechanisms for prediction: do insects have forward models? Trends Neurosci 27:278-282 
Weiss EJ, Flanders M (2004) Muscular and postural synergies of the human hand. J Neurophysiol 92:523-535

Weiss EJ, Flanders M (2011) Somatosensory comparison during haptic tracing. Cereb Cortex 21(2):425-434
Wu W, Gao Y, Bienenstock E, Donoghue JP, Black MJ (2006) Bayesian population decoding of motor cortical activity using a Kalman filter. Neural Comput 18:80-118 